\title{
$\mathrm{BMJ}$
}

\section{Total mortality after changes in leisure time physical activity in 50 year old men: 35 year follow-up of population based cohort}

\author{
Liisa Byberg, researcher , ${ }^{1}$ Håkan Melhus, professor , 2 Rolf Gedeborg, researcher , ${ }^{3}$ Johan Sundström, \\ researcher , ${ }^{4}$ Anders Ahlbom, professor , ${ }^{5}$ Björn Zethelius, researcher , ${ }^{6}$ Lars G Berglund, statistician , \\ Alicja Wolk, professor , ${ }^{8}$ Karl Michaëlsson, senior lecturer ${ }^{1}$
}

1Department of Surgical Sciences, Section of Orthopaedics, and Uppsala Clinical Research Centre, Uppsala University, SE-75185 Uppsala, Sweden

${ }^{2}$ Department of Medical Sciences, Section of Clinical Pharmacology, Uppsala University, Uppsala

${ }^{3}$ Department of Surgical Sciences, Section of Anaesthesiology and Intensive Care, and Uppsala Clinical Research Centre, Uppsala University, Uppsala

${ }^{4}$ Department of Medical Sciences, Section of Acute and Internal Medicine, Uppsala University, Uppsala

${ }^{5}$ Department of Epidemiology, Institute of Environmental Medicine, Karolinska Institutet, SE-17177 Stockholm, Sweden

${ }^{6}$ Department of Public Health and Caring Sciences, Section of

Geriatrics, Uppsala University, Uppsala

${ }^{7}$ Uppsala Clinical Research Centre, Uppsala University, Uppsala

${ }^{8}$ Department of Nutritional Epidemiology, Institute of

Environmental Medicine, Karolinska Institutet, Stockholm Correspondence to: L Byberg liisa.byberg@surgsci.uu.se

Cite this as: BMJ 2009;338:b688 doi:10.1136/bmi.b688

\section{ABSTRACT}

Objective To examine how change in level of physical activity after middle age influences mortality and to compare it with the effect of smoking cessation.

Design Population based cohort study with follow-up over 35 years.

Setting Municipality of Uppsala, Sweden.

Participants 2205 men aged 50 in 1970-3 who were reexamined at ages $60,70,77$, and 82 years.

Main outcome measure Total (all cause) mortality. Results The absolute mortality rate was $27.1,23.6$, and 18.4 per 1000 person years in the groups with low, medium, and high physical activity, respectively. The relative rate reduction attributable to high physical activity was $32 \%$ for low and $22 \%$ for medium physical activity. Men who increased their physical activity level between the ages of 50 and 60 continued to have a higher mortality rate during the first five years of follow-up (adjusted hazard ratio $2.64,95 \%$ confidence interval 1.32 to 5.27 , compared with unchanged high physical activity). After 10 years of follow-up their increased physical activity was associated with reduced mortality to the level of men with unchanged high physical activity $(1.10,0.87$ to 1.38$)$. The reduction in mortality associated with increased physical activity $(0.51,0.26$ to 0.97 , compared with unchanged low physical activity) was similar to that associated with smoking cessation $(0.64,0.53$ to 0.78 , compared with continued smoking).

Conclusions Increased physical activity in middle age is eventually followed by a reduction in mortality to the same level as seen among men with constantly high physical activity. This reduction is comparable with that associated with smoking cessation.

\section{INTRODUCTION}

Despite the known hazards of physical inactivity, it continues to be a major health problem. ${ }^{1}$ Physical inactivity is associated with increased incidence rates of obesity, diabetes, cardiovascular diseases, osteoporosis, and cancer. ${ }^{1-4}$ Short term randomised controlled trials in young to middle aged adults have shown a healthier risk profile with exercise. ${ }^{45}$ It is therefore recommended that adults engage in at least 30 minutes of moderate physical activity preferably on all days of the week. ${ }^{5-7}$ Adherence to these guidelines is associated with half the risk of mortality seen in sedentary people. ${ }^{8}$ Nevertheless, about half of all middle aged men in the West do not take part in regular physical activity. ${ }^{12}$ Whereas being physically inactive in younger years seems detrimental, we do not know whether an increase in exercise level later in life reduces mortality rates. If the impact on mortality could be compared with the effects of other changes in lifestyle habits it would be easier to communicate this potential health benefit.

In prospective studies that have examined changes in leisure time physical activity level in relation to mortality, ${ }^{9-15}$ few have considered the possibility of additional changes in physical activity behaviours during follow-up. ${ }^{13-15}$ Furthermore, the impact on mortality in relation to age at the time of the activity changes and the time elapsed since the changes occurred have not been established.

We investigated the impact of physical activity on total (all cause) mortality from middle to old age, taking into account changes in activity during follow-up. We studied this in a population based cohort of men aged 50 at baseline who were followed up for more than 35 years and investigated on five occasions. We also compared the effect of an increased level of physical activity on the mortality rate with that achieved by cessation of smoking.

\section{METHODS}

The present investigation used data from the population based Uppsala longitudinal study of adult men (ULSAM, www.pubcare.uu.se/ULSAM). Figure 1 shows the study population. In 1970 all 2841 men born in 1920-4 and living in the municipality of Uppsala, Sweden, were invited to attend a health survey (survey 1). ${ }^{16}$ Of those invited, 2322 men $(82 \%)$ aged 49-51 participated. Participants in survey 1 were invited for reinvestigations at ages 60, 70, and 77 


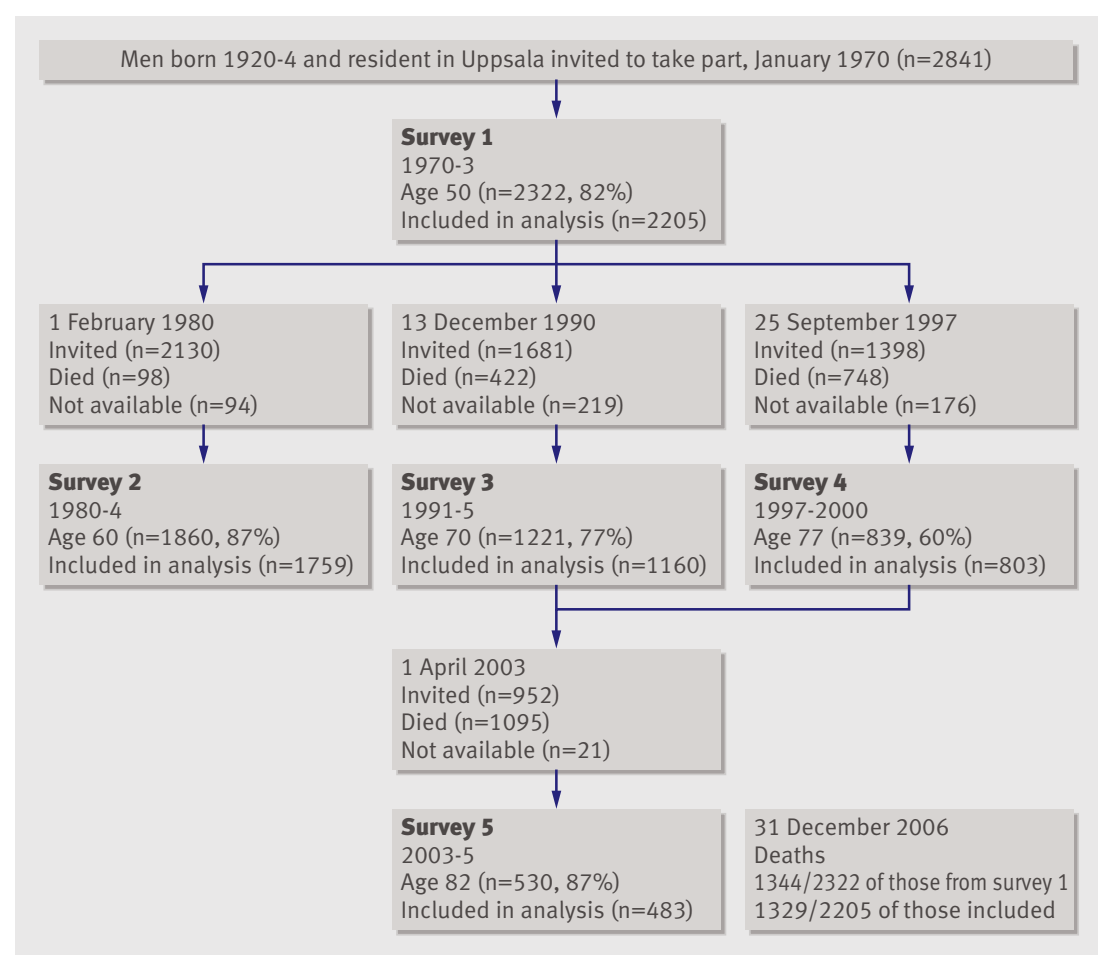

Fig 1 Cohort study base and numbers included in present study. Deaths presented as cumulative mortality from start of survey 1 . Numbers not available represent those who were not living in the Uppsala region at time of invitation. They did not contribute information on physical activity at that survey but they could return at later survey if they had moved back to Uppsala. Numbers included represent those who were included in analysis. All men were traced in population register for mortality data, including those "not available"

(surveys 2-4). Participants in surveys 3 and 4 were invited for reinvestigation at age 82 (survey 5).

We restricted our study population to the 2205 men who had responded to the questions regarding leisure time physical activity in the self administered questionnaire in survey 1 . Identical questions were asked at each survey:

1 Do you spend most of your time reading, watching TV, going to the cinema, or engaging in other, mostly sedentary activities?

2 Do you often go walking or cycling for pleasure?

3 Do you engage in any active recreational sports or heavy gardening at least 3 hours every week?

4 Do you regularly engage in hard physical training or competitive sport?

We considered the two highest activity categories, questions 3 and 4 , together as only $5 \%$ of the participants reported hard physical training. Participants answering yes to question 1 were categorised as low activity; 2 as medium; and 3 and 4 as high. The questionnaire has been used and validated in other studies and has also been shown to have a high reliability over time. ${ }^{17-20}$

Changes in the physical activity level between the first and second survey were considered in four categories: unchanged low (low or medium activity at both surveys), unchanged high (high activity at both surveys), decreased (high in survey 1, low or medium in survey 2), and increased (low or medium in survey 1 , high in survey 2).

Based on job titles retrieved from the national censuses from 1970 and 1980, we categorised occupational physical activity as sedentary, light, medium, high, unknown, and none (unemployed). ${ }^{21}$

Height and weight were measured at each survey and body mass index (BMI, weight $(\mathrm{kg}) /$ height $\left.(\mathrm{m})^{2}\right)$ calculated. Obesity was defined as BMI $\geq 30$. Systolic and diastolic blood pressure in the supine position was measured by standard procedures. Information on antihypertensive medication was retrieved by questionnaire, as was information on smoking habits. Smoking status was categorised as current smoker, former smoker, or never smoker. Changes in smoking status between two surveys were categorised as continued smoking, smoking cessation, and never smoking. Total fasting serum cholesterol concentration was measured with enzymatic techniques. The presence or absence of diabetes mellitus was determined at each survey according to the guidelines

Table 1| Sensitivity analysis, showing hazard ratios and $95 \%$ confidence intervals for each model

\begin{tabular}{|c|c|c|}
\hline $\begin{array}{l}\text { Physical activity } \\
\text { category }\end{array}$ & Model 1 & Model 2 \\
\hline \multicolumn{3}{|c|}{ Last observed value carried forward* } \\
\hline High & $1.00 \dagger$ & $1.00 \dagger$ \\
\hline Medium & $1.32(1.18$ to 1.49$)$ & 1.24 (1.10 to 1.39$)$ \\
\hline Low & 1.75 (1.48 to 2.06$)$ & 1.41 (1.19 to 1.67$)$ \\
\hline \multicolumn{3}{|c|}{ Lowest possible levelł } \\
\hline High & $1.00 \dagger$ & $1.00 \dagger$ \\
\hline Medium & 1.31 (1.16 to 1.48$)$ & $1.22(1.08$ to 1.38$)$ \\
\hline Low & $1.73(1.50$ to 2.00$)$ & $1.50(1.29$ to 1.74$)$ \\
\hline \multicolumn{3}{|c|}{ Highest possible level $\ddagger$} \\
\hline High & $1.00 \dagger$ & $1.00 \dagger$ \\
\hline Medium & 1.22 (1.09 to 1.37$)$ & $1.14(1.02$ to 1.29$)$ \\
\hline Low & 1.71 (1.45 to 2.02$)$ & $1.36(1.15$ to 1.62$)$ \\
\hline \multicolumn{3}{|c|}{ Excluding men with missing values§ } \\
\hline High & $1.00 \dagger$ & $1.00 \dagger$ \\
\hline Medium & 1.29 (1.14 to 1.46$)$ & 1.20 (1.06 to 1.36$)$ \\
\hline Low & 1.87 (1.57 to 2.23$)$ & $1.44(1.20$ to 1.72$)$ \\
\hline \multicolumn{3}{|c|}{ Excluding men with missing survey participationף } \\
\hline High & $1.00 \dagger$ & $1.00 \dagger$ \\
\hline Medium & 1.59 (1.32 to 1.92$)$ & $1.43(1.18$ to 1.74$)$ \\
\hline Low & 2.04 (1.56 to 2.65$)$ & $1.66(1.27$ to 2.17$)$ \\
\hline \multicolumn{3}{|c|}{$\begin{array}{l}\text { Model } 1 \text { includes physical activity as exposure. Model } 2 \text { includes } \\
\text { physical activity, smoking status, obesity status, height and weight } \\
\text { (both continuous); self perceived health (not good, good); physical } \\
\text { activity at work (sedentary, light, medium, high, unknown, unemployed); } \\
\text { diabetes mellitus, and any musculoskeletal, neurological, or psychiatric } \\
\text { disorders (yes, no for each disorder); and alcohol use (abstainer, } \\
\text { normal, suspected alcohol dependence). } \\
\text { * Missing values imputed by last observed value carried forward } \\
\text { method. } \\
\text { †Reference category. }\end{array}$} \\
\hline
\end{tabular}

fAdditional error was introduced to imputed values by assigning them lowest or highest possible level.

§Excludes all men with at least one missing observation; 5396 observations remained, representing 1918 participants and 1174 deaths; observation time was 52593 person years.

ףIncludes only men who attended all possible surveys before death 3188 observations remained, representing 895 participants and 525 deaths; observation time was 22814 person years. 
current at that time. Information on perceived health (classified as not good or good) and alcohol use at surveys 2 and 4 (classified according to the Michigan alcoholism screening test as abstainer, normal, suspected alcohol dependence ${ }^{22}$ ) was retrieved from the questionnaires. The census from 1970 provided information on educational level. Socioeconomic group at survey 1 was interview based and categorised as low, medium, and high. ${ }^{23}$ By linkage to the national hospital discharge register, we collected information on any musculoskeletal, neurological, or psychiatric disorder. The prevalence of each of these disorders and of diabetes was $<1.2 \%$ at baseline.

Using the individual 10 digit personal identification number given to all Swedish citizens, ${ }^{24}$ we traced the entire cohort in the continuously updated Swedish National Population Register until the date of death or 31 December 2006. Our main outcome was total (all cause) mortality. There was no loss to follow-up.

\section{Statistical analyses}

We used Cox's proportional hazards regression with delayed entry to estimate hazard ratios with 95\% confidence intervals and survival curves for the association between physical activity and mortality. The values of physical activity, as well as covariate information, were updated by data from each subsequent visit. The last observed value carried forward technique was used for missing data. The non-response frequencies for physical activity were $130,89,68$, and 0 at surveys $2-5$, respectively. Table 1 shows details of the sensitivity analyses.

Table 2 | Characteristics at baseline (survey 1 ) according to physical activity at age 50 . Values are means (SD) or number (percentage) of men

\begin{tabular}{|c|c|c|c|}
\hline \multirow[b]{2}{*}{ Variable and category } & \multicolumn{3}{|c|}{ Physical activity level } \\
\hline & Low $(n=325)$ & Moderate $(n=802)$ & High $(n=1078)$ \\
\hline Age (years) & $49.6(0.6)$ & $49.6(0.6)$ & $49.7(0.6)$ \\
\hline Weight (kg) & $78.7(12.8)$ & $77.3(11.1)$ & $77.8(10.6)$ \\
\hline Height (cm) & $175.9(6.1)$ & $176.0(6.0)$ & $176.5(5.8)$ \\
\hline Body mass index $\left(\mathrm{kg} / \mathrm{m}^{2}\right)$ & $25.4(3.7)$ & $24.9(3.2)$ & $25.0(3.1)$ \\
\hline No (\%) obese* & $40(12.3)$ & $41(5.1)$ & $62(5.8)$ \\
\hline Supine systolic blood pressure $(\mathrm{mm} \mathrm{Hg})$ & $133(19)$ & $134(18)$ & $132(17)$ \\
\hline Supine diastolic blood pressure $(\mathrm{mm} \mathrm{Hg})$ & $83(12)$ & $84(11)$ & $83(11)$ \\
\hline No (\%) taking antihypertensive drugs & $18(5.5)$ & $38(4.7)$ & $37(3.4)$ \\
\hline Total serum cholesterol (mmol/l) & $6.95(1.32)$ & $6.93(1.33)$ & $6.83(1.30)$ \\
\hline No (\%) with self rated good health & $242(74.5)$ & $641(79.9)$ & $902(83.7)$ \\
\hline \multicolumn{4}{|l|}{ No (\%) by smoking status: } \\
\hline Never smoker & $66(20.3)$ & $196(24.4)$ & $296(27.5)$ \\
\hline Former smoker & $61(18.8)$ & $181(22.6)$ & $281(26.1)$ \\
\hline Current smoker & 198 (60.9) & $425(53.0)$ & $501(46.5)$ \\
\hline \multicolumn{4}{|c|}{ No (\%) by occupational physical activity in 1970: } \\
\hline Sedentary & $33(10.2)$ & $86(10.7)$ & 139 (12.9) \\
\hline Light & $101(31.1)$ & $255(31.8)$ & $380(35.3)$ \\
\hline Medium & $39(12.0)$ & $100(12.5)$ & $126(11.7)$ \\
\hline High & $36(11.1)$ & $86(10.7)$ & $111(10.3)$ \\
\hline Unemployed/unknown & $116(35.7)$ & $275(34.3)$ & $322(29.9)$ \\
\hline
\end{tabular}
models were confirmed formally by Shoenfeld's tests, and graphically by comparing Nelson-Aalen plots. We considered one crude and two multivariable models. The first multivariable model included smoking status (current, former, never); weight and height (both continuous); self perceived health (good, not good); physical activity at work in 1970 and 1980 (sedentary, light, medium, high, unknown; or unemployed); alcohol use at ages 60 and 77 years (abstainer, normal, suspected alcohol dependence); and presence or absence of obesity, diabetes mellitus, and any musculoskeletal, neurological, or psychiatric disorder as covariates. The second multivariable model additionally included systolic and diastolic blood pressure, antihypertensive drugs, total serum cholesterol concentration, educational level, and socioeconomic group.

We assessed the relative excess risk due to interaction, a measure of biological interaction, ${ }^{25}$ from a Cox's proportional hazards model as described previously. $^{2627}$

Remaining life expectancy was calculated as the area under the survival curve using the trapezoidal rule approximation for the integral between the start of the study and the end of follow-up. We calculated differences in remaining life expectancy between exposure levels with $95 \%$ confidence intervals for the differences using the bootstrap centile method with 10000 replications.

In analyses of changes in physical activity level between the ages of 50 and 60, we partitioned follow-up time from date of examination in the second survey into five year intervals to account for non-proportional hazards.

Statistical analyses were performed with Stata/SE 10.1 (StataCorp, College Station, TX), SAS 9.1 (S AS Institute, Cary, NC, USA), and R (R Foundation for Statistical Computing, Vienna, Austria, 2007). 
Table 3 Hazard ratio (HR) and 95\% confidence intervals of total mortality for physical activity level in leisure time, smoking, and obesity status using time updated and baseline data

\begin{tabular}{|c|c|c|c|c|c|}
\hline \multirow[b]{2}{*}{ Exposure and category } & \multirow{2}{*}{$\begin{array}{l}\text { Time at risk } \\
\text { (person years) }\end{array}$} & \multirow[b]{2}{*}{ No of deaths } & \multicolumn{3}{|c|}{ Hazard ratio $(95 \% \mathrm{Cl})$} \\
\hline & & & Model 1 & Model 2 & Model 3 \\
\hline \multicolumn{6}{|l|}{ Time updated model* } \\
\hline \multicolumn{6}{|l|}{ Physical activity: } \\
\hline High & 28790 & 531 & $1.00 \dagger$ & $1.00 \dagger$ & $1.00 \dagger$ \\
\hline Medium & 25371 & 600 & 1.32 (1.18 to 1.49$)$ & 1.24 (1.10 to 1.39$)$ & 1.22 (1.08 to 1.37$)$ \\
\hline Low & 7306 & 198 & 1.75 (1.48 to 2.06$)$ & 1.41 (1.19 to 1.67$)$ & 1.40 (1.18 to 1.66$)$ \\
\hline \multicolumn{6}{|l|}{ Smoking status: } \\
\hline Never & 20657 & 465 & $1.00 \dagger$ & $1.00 \dagger$ & $1.00 \dagger$ \\
\hline Former & 19420 & 373 & 1.18 (1.02 to 1.36$)$ & 1.15 (1.00 to 1.32$)$ & $1.10(0.96$ to 1.27$)$ \\
\hline Current & 21389 & 491 & 2.09 (1.82 to 2.39) & 1.98 (1.72 to 2.28$)$ & 1.90 (1.64 to 2.19) \\
\hline \multicolumn{6}{|l|}{ Obesity status: } \\
\hline Non-obese & 56620 & 1157 & $1.00 \dagger$ & $1.00 \dagger$ & $1.00 \dagger$ \\
\hline Obese & 4847 & 172 & 1.52 (1.29 to 1.78$)$ & 1.49 (1.19 to 1.86$)$ & 1.51 (1.21 to 1.90$)$ \\
\hline \multicolumn{6}{|l|}{ Baseline model $\ddagger$} \\
\hline \multicolumn{6}{|l|}{ Physical activity: } \\
\hline High & 30930 & 617 & $1.00 \dagger$ & $1.00 \dagger$ & $1.00 \dagger$ \\
\hline Medium & 21850 & 504 & 1.20 (1.07 to 1.35$)$ & $1.16(1.03$ to 1.31$)$ & 1.14 (1.01 to 1.28$)$ \\
\hline Low & 8687 & 213 & 1.30 (1.12 to 1.52$)$ & 1.14 (0.98 to 1.34$)$ & $1.13(0.96$ to 1.33$)$ \\
\hline \multicolumn{6}{|l|}{ Smoking status: } \\
\hline Never & 16626 & 266 & $1.00 \dagger$ & $1.00 \dagger$ & $1.00 \dagger$ \\
\hline Former & 15446 & 291 & 1.21 (1.03 to 1.43$)$ & $1.22(1.03$ to 1.44$)$ & $1.20(1.01$ to 1.41$)$ \\
\hline Current & 29395 & 777 & 1.87 (1.63 to 2.16$)$ & 1.88 (1.63 to 2.17$)$ & 1.94 (1.67 to 2.24$)$ \\
\hline \multicolumn{6}{|l|}{ Obesity status: } \\
\hline Non-obese & 58032 & 1220 & $1.00 \dagger$ & $1.00 \dagger$ & $1.00 \dagger$ \\
\hline Obese & 3434 & 114 & $1.81(1.50$ to 1.20$)$ & 1.30 (1.01 to 1.68$)$ & $1.26(0.97$ to 1.62$)$ \\
\hline \multicolumn{6}{|c|}{$\begin{array}{l}\text { Model } 1 \text { includes one exposure only. Model } 2 \text { includes physical activity, smoking status, and obesity status; and as covariates height and weight (both } \\
\text { continuous); self perceived health (not good, good); physical activity at work (sedentary, light, medium, high, unknown, unemployed); diabetes } \\
\text { mellitus and any musculoskeletal, neurological, or psychiatric disorders (yes, no for each disorder); and alcohol use (abstainer, normal, suspected } \\
\text { alcohol dependence). Model } 3 \text { as for model } 2 \text { but with addition of systolic and diastolic blood pressure, use of antihypertensive drugs, total serum } \\
\text { cholesterol concentrations, educational level, and socioeconomic group. } \\
\text { ^Based on time updated data and mortality follow-up. } \\
\text { †Reference category. } \\
\ddagger \text { †Based on baseline data and mortality follow-up. }\end{array}$} \\
\hline
\end{tabular}

\section{RESULTS}

Table 2 shows baseline characteristics by level of physical activity at age 50. Almost half of the men reported a high level of physical activity, corresponding to at least three hours of recreational sports or heavy gardening a week, 36\% reported medium activity, corresponding to walks and cycling; and 15\% were sedentary. At the end of follow-up, we had 61456 person years and 1329 men $(60 \%)$ had died.

The absolute mortality rate was $27.1,23.6$, and 18.4 per 1000 person years for men with low, medium, and high physical activity. The relative rate reduction attributable to high physical activity was $32 \%$ compared with low activity and 22\% compared with medium activity. The relative mortality rates were highest among sedentary men and lowest among the most active men (table 3, fig 2). This trend was also apparent in each category of smoking (not shown). The estimated effect of sedentary behaviour on mortality was similar to the effect of smoking and obesity (table 3). Adjustment for covariates attenuated the associations.
The models in which we used time updated variables showed higher estimates of the effect of physical activity than models with baseline data (table 3).

We converted the differences in death rates into differences in remaining life expectancies from age 50 . Men who reported high levels of physical activity were expected to live 3.8 (95\% confidence interval 2.7 to 4.8 ) years longer than sedentary men and 1.8 (1.3 to 2.2 ) years longer than men who reported medium levels of physical activity. After adjustment for potential confounding variables the corresponding remaining life expectancies were 2.3 (1.3 to 3.3) and 1.1 (0.6 to 1.6) years, respectively.

\section{Changes in physical activity level}

We investigated the influence on mortality of changes in physical activity between the ages of 50 and 60 in the 1759 men who participated in the first and second survey. From the date of examination in survey 2, we studied 34020 person years and 998 men died. At the age of 60 about $27 \%(\mathrm{n}=474)$ still reported high physical activity, $11 \%(\mathrm{n}=194)$ had increased and $24 \%(\mathrm{n}=414)$ had reduced levels, and 38\% $(\mathrm{n}=677)$ reported 
unchanged low activity. Men who changed their physical activity level could not be distinguished from men with unchanged activity based on baseline clinical characteristics (not shown).

Changes in physical activity between the ages of 50 and 60 were associated with mortality (fig 3). After 10 years of follow-up the mortality rate did not differ between men who had increased their physical activity and men who were at an unchanged high level (table 4). This is also illustrated by the parallel failure curves after about age 70 (fig 3). During the first five years of follow-up, however, the mortality rate was 2.6 times higher in men who had increased their level of physical activity compared with men whose physical activity was high but unchanged (table 3). Adjustment for smoking, obesity, and other covariates attenuated the estimates but did not change the interpretation of the results.

Eighteen of the 154 deaths (12\%) among men with increased physical activity occurred within the first five years of follow-up and 21 men died during the subsequent five years. This group with increased mortality during the first 10 years after an increase in physical activity could not be distinguished on the basis of differences in the cardiovascular risk factor profile either at age 50 or 60 . Exclusion of men with diagnosis of or echocardiography signs of cardiovascular disease at the age of 50 or 60 did not change any of the observed associations. Neither was there any difference in causes of death between this group and others. Men who maintained a low level of physical activity and men who decreased their level had higher mortality rates

\footnotetext{
— Unchanged low or medium physical activity

- - - Decreased from high to low or medium physical activity

----- Increased from low or medium to high physical activity

-- Unchanged high physical activity
}

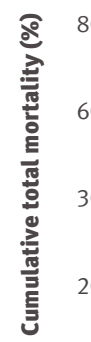

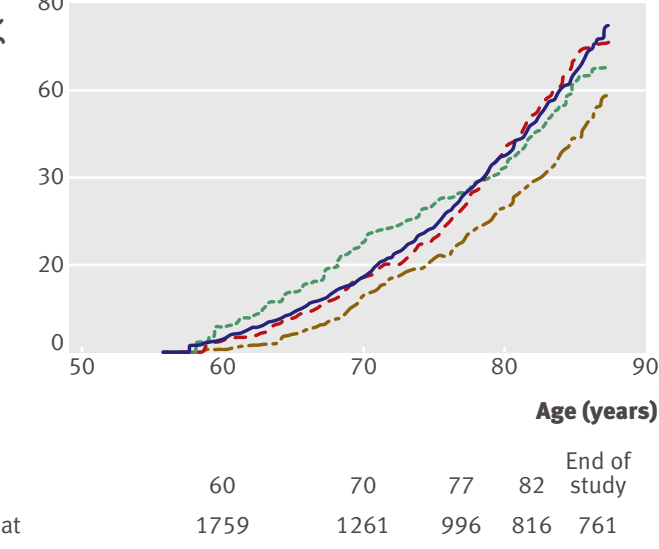

1759

$1261996 \quad 816 \quad 761$

Fig 3 | Cumulative mortality from age 60 (Kaplan-Meier) according to changes in leisure time physical activity level and total mortality. Slopes of curves correspond to mortality rates. Parallel curves indicate equal rates of death. At the end of follow-up, rates were 74.0 (95\% confidence interval 67.0 to 80.6) for unchanged low-medium, 70.5 (64.8 to 76.0) for decreased from high to low-medium, 64.7 (57.4 to 71.9 ) for increased from low-medium to high, and 58.5 (50.8 to 66.3) for unchanged high than those with unchanged high physical activity, irrespective of the length of follow-up (table 4, fig 3).

We studied men who increased their physical activity level in more detail. The mortality rate after more than 10 years of follow-up was halved in men who had increased their physical activity to a high level both from a medium (adjusted hazard ratio 0.58, 0.39 to $0.87)$ and a low $(0.51,0.26$ to 0.97$)$ level. There was no evident reduction in mortality rate among men who increased their physical activity from low to medium (0.81, 0.51 to 1.31$)$. As a comparison, smoking cessation was associated with a $40 \%$ lower mortality rate after more than 10 years of follow-up compared with current smoking $(0.64,0.53$ to 0.78$)$. Men who had never smoked had a $60 \%$ lower mortality rate than current smokers $(0.42,0.33$ to 0.52$)$.

There was no evidence of biological interaction between increased physical activity and smoking cessation in their effects on mortality; the relative excess risk due to interaction was -0.40 ( -1.30 to 0.50$)$.

\section{DISCUSSION}

Principal findings

Increased levels of physical activity in middle age have an effect on mortality. After a 10 year period of increased physical activity the excess mortality seen in inactive men was reduced to the same levels of mortality as seen in physically active men. There is, however, a period of at least 5 years before this risk reduction during which the risk is higher. The halved mortality rate after 10 years of follow-up after increased physical activity between the ages of 50 and 60 (compared with continued inactivity) was similar to that seen after smoking cessation (compared with continued smoking).

\section{Strengths and weaknesses of the study}

The main strengths of our study include the population based design, the long follow-up from a time before the men reached ages with a high mortality rate, the repeated measurements, and the objective and complete information on mortality. Our analyses also took into account changes over time in potential confounders including smoking, obesity, self perceived health, and morbidity and in classic risk factors for mortality including hypertension and total cholesterol concentration. We also adjusted our estimates for socioeconomic group and educational level.

The main limitation of our study is its restriction to men and the crude assessment of physical activity by questionnaire, with risk of misclassification possibly leading to underestimation of the results. ${ }^{28}$ There is, however, no ideal test to measure physical activity, ${ }^{29}$ either by questionnaire or by more objective assessments. We used a questionnaire created in collaboration with the Swedish National Institute of Public Health that estimates physical activity over the life cycle. ${ }^{30}$ Although our categories indexing physical activity should be regarded as reflecting common perceptions or patterns of physical activity rather than precise measures of levels, the levels estimated from 
Table 4 | Hazard ratios with $95 \%$ confidence intervals for total mortality after different follow-up periods by changes in physical activity between ages of 50 and 60

\begin{tabular}{|c|c|c|c|c|}
\hline \multirow{2}{*}{$\begin{array}{l}\text { Follow-up period and physical activity } \\
\text { level }\end{array}$} & \multirow{2}{*}{$\begin{array}{c}\text { Follow-up } \\
\text { (person years) }\end{array}$} & \multirow{2}{*}{$\begin{array}{l}\text { No of } \\
\text { deaths }\end{array}$} & \multicolumn{2}{|c|}{ Hazard ratio $(95 \% \mathrm{Cl})$} \\
\hline & & & Model 1 & Model 2 \\
\hline \multicolumn{5}{|l|}{ Unrestricted } \\
\hline Unchanged high & 10508 & 249 & $1.00^{*}$ & $1.00^{*}$ \\
\hline Increased from low or moderate to high & 4862 & 154 & 1.27 (1.04 to 1.55$)$ & 1.21 (0.99 to 1.48$)$ \\
\hline Reduced from high to low or moderate & 7020 & 233 & 1.53 (1.28 to 1.83$)$ & 1.35 (1.12 to 1.61 \\
\hline Unchanged low or moderate & 11629 & 362 & $1.47(1.25$ to 1.73$)$ & $1.33(1.12$ to 1.56 \\
\hline \multicolumn{5}{|l|}{ Deaths after 5 years of follow-up } \\
\hline Unchanged high & 10464 & 234 & $1.00^{*}$ & $1.00^{\star}$ \\
\hline Increased from low or moderate to high & 4813 & 136 & $1.18(0.95$ to 1.45$)$ & $1.13(0.91$ to 1.39$)$ \\
\hline Reduced from high to low or moderate & 6952 & 211 & $1.51(1.25$ to 1.82$)$ & $1.33(1.10$ to 1.61 \\
\hline Unchanged low or moderate & 11542 & 325 & $1.46(1.23$ to 1.73$)$ & 1.31 (1.11 to 1.56 \\
\hline \multicolumn{5}{|l|}{ Deaths after 10 years of follow-up } \\
\hline Unchanged high & 10190 & 198 & $1.00^{*}$ & $1.00^{*}$ \\
\hline Increased from low or moderate to high & 4649 & 115 & $1.14(0.90$ to 1.43$)$ & 1.10 (0.87 to 1.38$)$ \\
\hline Reduced from high to low or moderate & 6694 & 178 & $1.60(1.30$ to 1.96$)$ & $1.43(1.17$ to 1.76 \\
\hline Unchanged low or moderate & 11064 & 263 & 1.49 (1.24 to 1.79$)$ & $1.36(1.13$ to 1.64 \\
\hline \multicolumn{5}{|l|}{ Deaths within 5 years of follow-up } \\
\hline Unchanged high & 2340 & 15 & $1.00^{*}$ & $1.00^{\star}$ \\
\hline Increased from low or moderate to high & 929 & 18 & 3.05 (1.54 to 6.06$)$ & $2.64(1.32$ to 5.27$)$ \\
\hline Reduced from high to low or moderate & 2028 & 22 & $1.66(0.86$ to 3.20$)$ & 1.50 (0.77 to 2.92$)$ \\
\hline Unchanged low or moderate & 3287 & 37 & 1.75 (0.96 to 3.19$)$ & $1.58(0.86$ to 2.90$)$ \\
\hline \multicolumn{5}{|l|}{ Deaths within 10 years of follow-up } \\
\hline Unchanged high & 4589 & 51 & $1.00^{*}$ & $1.00^{*}$ \\
\hline Increased from low or moderate to high & 1783 & 39 & 1.98 (1.30 to 3.00$)$ & $1.70(1.12$ to 2.60$)$ \\
\hline Reduced from high to low or moderate & 3874 & 55 & 1.25 (0.86 to 1.83$)$ & 1.10 (0.74 to 1.61$)$ \\
\hline Unchanged low or moderate & 6326 & 99 & $1.43(1.02$ to 2.01$)$ & $1.23(0.87$ to 1.74$)$ \\
\hline
\end{tabular}

Model 1 includes time updated variable change in physical activity as exposure. Model 2 includes time updated variable change in physical activity as exposure and covariates: smoking status, obesity status, height and weight, self perceived health, physical activity at work, diabetes mellitus, any musculoskeletal, neurological, or psychiatric disorders, and alcohol use.

*Reference category.

these four simple questions are positively associated with physiological measurements of fitness, ${ }^{16}{ }^{17}$ skeletal muscle capillary density, ${ }^{31}$ and pedometer results, ${ }^{32}$ indicating that these levels do represent physical activity. Simple questionnaires are well suited for the study of changed behaviour in large epidemiological studies, ${ }^{28}$ and the repetition of identical questions at each survey is one of the main strengths of our study.

The last observed value carried forward technique could introduce bias, despite being the preferred imputation method. ${ }^{33}$ As results from sensitivity analysis were consistent with the main results, it seems unlikely that this imputation method introduced bias. In our multivariable models, the adjustment for variables that can be regarded not only as confounders but also as intermediates on the causal pathway - such as perceived health, body weight, and diabetes - might also have conservatively biased our estimates.

Strengths and weaknesses in relation to other studies Many previous studies have used baseline data and changes in activity to examine whether physical activity influences mortality, without applying a time dependent approach in their analyses..$^{9-123435}$ Our associations between baseline data on physical activity and mortality rates were generally weaker than when we took into account changes within individuals in the level of physical activity during follow-up, further emphasising the importance of using time updated variables when behaviour such as physical activity is studied in relation to clinical outcomes. ${ }^{36}$ Time dependent life table analysis for estimation of remaining life expectancy among 50 year old men in the Framingham Heart Study showed 3.9-4.1 years longer life expectancy for men reporting high physical activity and 1.6-1.8 years longer life expectancy for men reporting medium physical activity, compared with the sedentary group. ${ }^{1314}$ We obtained estimates of similar magnitude.

Total mortality was substantially influenced by the length of time since the change in physical activity took place. One recent study that used time dependent analysis to investigate change in physical activity in relation to mortality in men and women aged 19 to over 90 years found an association between change in physical activity and mortality in men under the age of $70 .{ }^{15}$ In that study, however, the direction of change, the age when the change in physical activity occurred, and the time that had elapsed since the change remained unclear.

A change in physical activity in the lower activity range might reflect a change in sedentary behaviour rather than in actual physical activity. Recent research showed that sedentary behaviour and physical activity were independently associated with obesity. ${ }^{37}$ Future research should aim to separate these two effects.

As measurement of physical activity is crude and difficult to compare between studies, we wanted to compare the effect of physical activity on mortality with the effect of smoking. Self reported smoking has a high validity, ${ }^{38}$ and smoking is probably the most well established modifiable behavioural risk factor for premature death. ${ }^{39}$ The graded inverse association between physical activity level and mortality was independent of and present in each category of smoking status. The impact of increased physical activity on mortality was on par with the effect of smoking cessation. A recent study in women found a comparable reduction in mortality after smoking cessation. $^{40}$

Our observational results might differ from those from meta-analyses of clinical trials because such trials tend to be conducted in high risk populations and are designed so that the exposure has a low measurement error and that the probability of confounding is reduced. The effect we observed after increased physical activity, however, is larger than the effect of a $1 \mathrm{mmol} / \mathrm{l}$ reduction in cholesterol concentration $(18 \%$ reduction of total mortality $)^{41}$ and the effect of treatment with any antihypertensive drug $(10 \%$ reduction of total mortality). ${ }^{42}$

\section{Possible explanations}

We previously showed that an increase in the level of physical activity in the present cohort was associated with beneficial changes in the metabolic profile, including a decrease in body weight and in blood 


\section{WHAT IS ALREADY KNOWN ON THIS TOPIC}

Physical activity is beneficial for health and prolongs life among younger individuals

Inactivity and resulting disease remains a major health problem

\section{WHAT THIS STUDY ADDS}

There is a dose dependent association between level of physical activity and total mortality in middle aged men

Increased physical activity prolongs life among middle aged and older men, though there might be a period of 5-10 years before an effect is seen on total mortality

The effect on mortality after increased physical activity is on par with that of smoking cessation

concentrations of insulin, proinsulin, glucose, and lipids. ${ }^{43}$ An improved metabolic profile reduces the prevalence of metabolic syndrome, obesity, and cardiovascular disease. This is one suggested pathway whereby physical activity is thought to reduce mortality. ${ }^{1334}$ The effect of hypertension and high total cholesterol concentration on mortality is well established, but in our analysis the association between physical activity and mortality was independent of these. Other suggested mechanisms for the effect of physical activity on mortality include changes in inflammatory and hormonal responses and gut mobility ${ }^{44}$ and effects on the nervous systems, which influence neuromuscular and brain function. ${ }^{45}$ All these mechanisms might be important for the progression of many diseases and general wellbeing. In older people physical activity is also important for maintaining muscle strength, postural balance, ${ }^{4647}$ functional independence, and health related quality of life. ${ }^{48}$ The effect of physical activity on the risk of premature death might be modulated by genetic differences. ${ }^{49}$ Recent results in Swedish twins, however, argue against genetics as a cause of the association between physical activity and mortality. ${ }^{50}$ In addition, inactive twins have been reported to have shorter telomere lengths corresponding to higher biological age by up to 10 years compared with their more active co-twin. ${ }^{51}$

Implications, unanswered questions, and future research Within the first five years after the investigation at the age of 60 we observed an increased risk associated with increased physical activity compared with men with unchanged high activity. Given the small numbers of deaths we are reluctant to place a strong emphasis on this increased risk, especially as mortality was not higher than mortality in men who continued to be sedentary. Although there might not be a true increase in mortality soon after increased physical activity, there is nonetheless no immediate benefit. This has also been observed in a community based cluster randomised trial in older people in whom participation in an exercise programme had no effect on mortality during the two year follow-up. ${ }^{52}$ There was, however, a much larger than expected improvement in health related quality of life after the intervention, suggesting that increased activity might have early health benefits that are collectively too small to have an impact on total mortality.

Our results could have important implications in the planning of clinical trials as these would need to be long enough to extend beyond the period of no benefit and to take into consideration the ethical implications of a possible increase in short term risk. Different follow-up times might also yield different results in observational studies.

It is difficult to quantify the least amount of physical activity needed for a beneficial effect on mortality in our study. Our dose-response relations suggest that the more activity the better, but on the basis of our data it is not possible to determine whether the recommended minimum amount of physical activity was achieved or not. $^{5-7}$

We could not identify any characteristics distinguishing those who died shortly after taking up physical activity. A healthy survivor effect cannot be excluded as survival until age 60 was necessary for this analysis. Further research should investigate whether and to what extent increased physical activity affects mortality in the period soon after the change. In addition, the effects in other age groups and in women need to be studied. Mechanisms and reasons for an active choice to change physical activity are not fully understood.

\section{Conclusion}

In conclusion, we have shown a graded reduction in total mortality risk with increasing physical activity level in men. Increased physical activity in middle age increases longevity after an induction period of up to 10 years of no benefit. An increase in physical activity has the same impact on lowering mortality rate in the long term as smoking cessation. Thus, efforts for promotion of physical activity, even among middle aged and older men, are important.

Contributors: LB and KM designed the study, analysed and interpreted the data, wrote the manuscript, and are guarantors. HM contributed to the study design, interpretation of the data, and writing of the manuscript. LGB provided statistical advice, analysed and interpreted the data, and commented on drafts of the manuscript. RG and AA interpreted the data and made significant contributions to drafts of the manuscript. JS, BZ, and AW provided clinical and methodological advice and commented on drafts of the manuscript. All authors declare that they accept full responsibility for the conduct of the study, had access to the data, and controlled the decision to publish

Funding: The study was funded by the Swedish Research Council. The funder had no role in the design and conduct of the study; the collection, management, analysis, and interpretation of data; the writing of the manuscript; or the decision to submit the manuscript for publication. Competing interests: None declared.

Ethical approval: The study was approved by the ethics committee at the Faculty of Medicine, Uppsala University, Sweden, and all participants gave written informed consent

1 Bull FC, Armstrong TP, Dixon T, Ham S, Neiman A, Pratt M. Physical inactivity. In: Ezzati M, Lopez AD, Rodgers A, Murray CJL, eds. Comparative quantification of health risks. Global and regional burden of disease attributable to selected major risk factors. Geneva: World Health Organization,

2004:729-882. www.who.int/publications/cra/chapters/volume1/ 0729-0882.pdf.

2 Boström G. Habits of life and health. Scand J Public Health 2006;34:199-228.

3 Farahmand BY, Ahlbom A, Ekblom Ö, Ekblom B, Hållmarker U, Aronson D, et al. Mortality amongst participants in Vasaloppet: a 
classical long-distance ski race in Sweden. J Intern Med 2003;253:276-83.

4 Lakka TA, Laaksonen DE. Physical activity in prevention and treatmen of the metabolic syndrome. Appl Physiol Nutr Metab 2007;32:76-88.

5 Pate RR, Pratt M, Blair SN, Haskell WL, Macera CA, Bouchard C, et al. Physical activity and public health. A recommendation from the Centers for Disease Control and Prevention and the American College of Sports Medicine. JAMA 1995;273:402-7.

6 Haskell WL, Lee I-M, Pate RR, Powell KE, Blair SN, Franklin BA, et al. Physical activity and public health: updated recommendation for adults from the American College of Sports Medicine and the American Heart Association. Circulation 2007;116:1081-93.

7 Physical Activity Guidelines Advisory Committee. Physical activity guidelines advisory committee report. Washington, DC: US Department of Health and Human Services,

2008. www.health.gov/paguidelines/Report/Default.aspx.

8 Leitzmann MF, Park Y, Blair A, Ballard-Barbash R, Mouw T, Hollenbeck AR, et al. Physical activity recommendations and decreased risk of mortality. Arch Intern Med 2007;167:2453-60.

9 Katzmarzyk PT, Janssen I, Ardern Cl. Physical inactivity, excess adiposity and premature mortality. Obes Rev 2003;4:257-90.

10 Paffenbarger Jr RS, Hyde RT, Wing AL, Lee IM, Jung DL, Kampert JB. The association of changes in physical-activity level and other lifestyle characteristics with mortality among men. N Engl J Med 1993;328:538-45

11 Wannamethee S, Shaper A, Walker M. Changes in physical activity, mortality, and incidence of coronary heart disease in older men. Lancet 1998;351:1603-8.

12 Schnohr P, Scharling H, Jensen JS. Changes in leisure-time physical activity and risk of death: an observational study of 7,000 men and women. Am J Epidemiol 2003;158:639-44.

13 Franco $\mathrm{OH}$, de Laet C, Peeters A, Jonker J, Mackenbach J, Nusselder W. Effects of physical activity on life expectancy with cardiovascular disease. Arch Intern Med 2005;165:2355-60.

14 Jonker JT, De Laet C, Franco OH, Peeters A, Mackenbach J, Nusselder WJ. Physical activity and life expectancy with and without diabetes: life table analysis of the Framingham Heart Study. Diabetes Care 2006;29:38-43.

15 Talbot LA, Morrell CH, Fleg JL, Metter EJ. Changes in leisure time physical activity and risk of all-cause mortality in men and women: the Baltimore Longitudinal Study of Aging. Prev Med 2007;45:169-76.

16 Michaëlsson K, Olofsson H, Jensevik K, Larsson S, Mallmin H, Berglund $\mathrm{L}$, et al. Leisure physical activity and the risk of fracture in men. PLoS Medicine 2007; 4:e199.

17 Løchen M-L, Rasmussen K. The Tromsø study: physical fitness, self reported physical activity, and their relationship to other coronary risk factors. J Epidemiol Community Health 1992;26:103-7.

18 Rosengren A, Wilhelmsen L. Physical activity protects against coronary death and deaths from all causes in middle-aged men. Evidence from a 20-year follow-up of the primary prevention study in Göteborg. Ann Epidemiol 1997;7:69-75.

19 Collen MF, Cutler JL, Siegelaub AB, Cella RL. Reliability of a selfadministered medical questionnaire. Arch Intern Med 1969;123:664-81.

20 Aires N, Selmer R, Thelle D. The validity of self-reported leisure time physical activity, and its relationship to serum cholesterol, blood pressure and body mass index. A population based study of 332,182 men and women aged 40-42 years. Eur J Epidemiol 2003;18:479-85.

21 Farahmand BY, Persson P-G, Michaëlsson K, Baron JA, Alberts A, Moradi T, et al. Physical activity and hip fracture: a population-based case-control study. Int J Epidemiol 2000;29:308-14.

22 Selzer ML. The Michigan alcoholism screening test: the quest for a new diagnostic instrument. Am J Psychiatry 1971;127:1653-8.

23 Andersen R, Smedby B, Anderson D. Medical care use in Sweden and the United States. A comparative analysis of systems and behaviour. Chicago: Center for Health Administration Studies, 1970.

24 Calltorp J, Adami HO, Åström H, Fryklund L, Rössner S, Trolle Y, et al. Country profile: Sweden. Lancet 1996;347:587-94.

25 Rothman KJ. Modern epidemiology. Philadelphia: Lippincott Williams and Wilkins, 1998

26 Andersson T, Alfredsson L, Källberg H, Zdravkovic S, Ahlbom A Calculating measures of biological interaction. Eur J Epidemiol 2005;20:575-9.

27 Li R, Chambless L. Test for additive interaction in proportional hazards models. Ann Epidemiol 2007;17:227-36.

28 Shephard RJ. Limits to the measurement of habitual physical activity by questionnaires. Br J Sports Med 2003;37:197-206.

29 Ferrari $\mathrm{P}$, Friedenreich C, Matthews CE. The role of measurement error in estimating levels of physical activity. Am J Epidemiol 2007;166:832-40
30 Saltin B, Grimby G. Physiological analysis of middle-aged and old former athletes. Comparison with still active athletes of the same ages. Circulation 1968;38:1104-15

31 Hedman A, Byberg L, Reneland R, Lithell HO. Muscle morphology, selfreported physical activity and insulin resistance syndrome. Acta Physiol Scand 2002;175:325-32.

32 Sequeira MM, Rickenbach M, Wietlisbach V, Tullen B, Schutz Y. Physical activity assessment using a pedometer and its comparison with a questionnaire in a large population survey. Am J Epidemiol 1995;142:989-99.

33 Engels JM, Diehr P. Imputation of missing longitudinal data: a comparison of methods. J Clin Epidemiol 2003;56:968-76

34 Erlichman J, Kerbey AL, James WPT. Physical activity and its impact on health outcomes. Paper 1: the impact of physical activity on cardiovascular disease and all-cause mortality: an historical perspective. Obes Rev 2002;3:257-71.

35 Gregg EW, Cauley JA, Stone K, Thompson TJ, Bauer DC, Cummings SR, et al. Relationship of changes in physical activity and mortality among older women. JAMA 2003;289:2379-86.

36 Andersen LB. Relative risk of mortality in the physically inactive is underestimated because of real changes in exposure level during follow-up. Am J Epidemiol 2004;160:189-95

37 Hamilton MT, Hamilton DG, Zderic TW. Role of low energy expenditure and sitting in obesity, metabolic syndrome, type 2 diabetes, and cardiovascular disease. Diabetes 2007:56:2655-67.

38 Vartiainen E, Seppälä T, Lillsunde P, Puska P. Validation of self reported smoking by serum cotinine measurement in a communitybased study. J Epidemiol Community Health 2002;56:167-70.

39 Doll R, Peto R, Boreham J, Sutherland I. Mortality in relation to smoking: 50 years' observations on male British doctors. BMJ 2004;328:1519.

40 Kenfield SA, Stampfer M], Rosner BA, Colditz GA. Smoking and smoking cessation in relation to mortality in women. JAMA 2008;299:2037-47.

41 Gould AL, Davies GM, Alemao E, Yin DD, Cook JR. Cholesterol reduction yields clinical benefits: meta-analysis including recent trials. Clin Ther 2007;29:778-94.

42 Psaty BM, Lumley T, Furberg CD, Schellenbaum G, Pahor M, Alderman $\mathrm{MH}$, et al. Health outcomes associated with various antihypertensive therapies used as first-line agents: a network metaanalysis. JAMA 2003;289:2534-44.

43 Byberg L, Zethelius B, McKeigue PM, Lithell HO. Changes in physical activity are associated with changes in metabolic cardiovascular risk factors. Diabetologia 2001;44:2134-9.

44 Friedenreich CM, Orenstein MR. Physical activity and cancer prevention: etiologic evidence and biological mechanisms. J Nutr 2002;132:3456-64S.

45 Dishman RK, Berthoud HR, Booth FW, Cotman CW, Edgerton VR, Fleshner MR, et al. Neurobiology of exercise. Obesity 2006;14:345-56.

46 Kuh D, Bassey EJ, Butterworth S, Hardy R, Wadsworth MEJ, and the Musculoskeletal Study Team. Grip strength, postural control, and functional leg power in a representative cohort of British men and women: associations with physical activity, health status, and socioeconomic conditions. J Gerontol A Biol Sci Med Sci 2005;60:224-31.

47 Portegijs E, Rantanen T, Sipilä S, Laukkanen P, Heikkinen E. Physical activity compensates for increased mortality risk among older people with poor muscle strength. Scand J Med Sci Sports 2006;17:473-9.

48 Tessier S, Vuillemin A, Bertrais S, Boini S, Le Bihan E, Oppert JM, et al. Association between leisure-time physical activity and health-related quality of life changes over time. Prev Med 2007;44:202-8.

49 Rankinen T, Bouchard C. Invited commentary: physical activity, mortality, and genetics. Am J Epidemiol 2007;166:260-2.

50 Carlsson S, Andersson T, Lichtenstein P, Michaëlsson K, Ahlbom A. Physical activity and mortality: is the association explained by genetic selection? Am J Epidemiol 2007;166:255-9.

51 Cherkas LF, Hunkin JL, Kato BS, Richards JB, Gardner JP, Surdulescu GL, et al. The association between physical activity in leisure time and leukocyte telomere length. Arch Intern Med 2008;168:154-8.

52 Munro JF, Nicholl JP, Brazier JE, Davey R, Cochrane T. Cost effectiveness of a community based exercise programme in over 65 year olds: cluster randomised trial. J Epidemiol Community Health 2004;58:1004-10.

Accepted: 16 December 2008 